\title{
The current genomic approaches for common respiratory diseases
}

\begin{abstract}
Common respiratory diseases including asthma, chronic obstructive pulmonary disease (COPD) and lung cancer are increasing worldwide. Genomic approaches for the diseases provided new insights of the mechanisms and revealed the new therapeutic targets. Genome-wide association studies (GWAS), epigenetic studies, transcriptional profiling, RNA sequencing, chromatin immune precipitation (ChIP) sequencing and microbiota investigation make personalized medicine for common respiratory diseases possible in the near future. In this review, we summarize the current progress of genomic approaches for asthma, COPD and lung cancer and also discuss the future directions of the research of the diseases.
\end{abstract}

Keywords: genomic, asthma, chronic obstructive pulmonary diseases, lung cancer
Volume 3 Issue 3 - 2016

\author{
Shijun Lin,' Youming Zhang, ${ }^{2}$ Min Zhang ${ }^{3}$ \\ 'Department of Thorax Medicine, Lung gang People's Hospital, \\ PR China \\ 2Molecular Genetics Group, Division of Respiratory Sciences, \\ Imperial College London, UK \\ ${ }^{3}$ Department of Thorax Medicine, the First Affiliated Hospital of \\ Shenzhen University, China
}
Correspondence: Min Zhang, Department of Thorax Medicine, the First Affiliated Hospital of Shenzhen University, Shenzhen, PR China, Email hi_zm@I63.com Youming Zhang, Molecular Genetics Group, National Heart and Lung Institute, Dovehouse Street, London SW3 6LY, Email y.zhang@imperial.ac.uk

Received: October 26, 2016 | Published: November 10, 2016

\begin{abstract}
Abbreviations: COPD, chronic obstructive pulmonary disease; GWAS, genome-wide association studies; ER, endoplasmic reticulum; IREs, iron-responsive elements; SCLC, small cell lung cancer; NSCLC, non-small cell lung cancer; ZFN, zinc finger nuclease; RAGE, receptor for advanced glycan end products; CRISPER, clustered regularly interspaced short palindromic repeats
\end{abstract}

\section{Introduction}

The burden of chronic respiratory diseases and lung cancer is rapidly increasing worldwide. Human common respiratory diseases are complex disorders underlined by a broad range of genetic and environmental factors. Asthma and chronic obstructive pulmonary disease (COPD) are the most common respiratory diseases that are characterized by inflammation of the small airways of the lung with symptoms of wheeze and shortness of breath. The presence of inflammation can lead to airway scarring and intractable airflow limitation. Lung cancer is the leading cause of cancer death around the world. The death numbers of patients from lung cancer in America every year were greater than the death numbers of patients from prostate, breast, and colon cancer combined. ${ }^{1}$ In recent years, with the development of genetics and genomics approaches, personalized medicine becomes more and more available for patients who have chronic respiratory diseases and lung cancers in clinic. The systemic approaches with genome-wide association studies, epigenetic studies, transcriptional profiling, RNA sequencing, ChIP sequencing and microbiota investigation brought a huge influx of data to chronic respiratory diseases and lung cancer. In this review, we briefly introduce the methodologies for genomic approaches and summarize the current progress in asthma, COPD and lung cancer, we also discuss the future research directions of the diseases.

\section{The genomic approach methodologies}

GWAS is a powerful approach for identifying the genetic causes of complex traits such as asthma and COPD. It examines the relationships between allele frequencies and disease status with a large number of genetic markers across the genome. It is efficient to investigate the entire genome simultaneously. GWAS provides the opportunity to identify novel mechanisms of disease pathogenesis that are caused by previously unsuspected genes or regulatory regions. More than 2,000 associations for more than 300 complex diseases and traits were identified in recent years by GWASs. ${ }^{2}$ The strength of GWAS is that many novel genes that were never suspected to have roles in the diseases were identified. Epigenetic effect is other possible cause of familial clustering. It regulates gene expression that is not caused by changes in the DNA sequence but by DNA methylation, histone modification and other mechanisms. The patterns of gene expression that determine cellular types and function are due to methylation of $\mathrm{CpG}$ sequences and gene silencing. Sex, age and other environmental factors have all been strongly associated with altered methylation in the genome. Histones are highly alkaline proteins in eukaryotic cell nuclei that package the DNA into nucleosome. Methylation, acetylation, phosphorylation, ubiquitination and sumoylation are major histone modifications. The modification can affect specific genes from activation to inactivation or vice versa. Transcriptional profiling is to quantify the transcriptional level (RNA level) of the genome in cells or tissues. There are two methods for systematic quantification of RNA in cells or tissues; one is microarray systems and the other is RNA sequencing.

The advantage for RNA sequencing is that it can possibly identify the regulation non-coding RNA in the relevant cells or tissues. Noncoding RNAs (ncRNAs) emerged as novel molecules that are important in human diseases in recent years. ncRNAs include housekeeping RNAs, long noncoding RNAs and small noncoding RNAs. Micro RNAs (miRNAs) are the most studied small ncRNAs. miRNAs are about 18-25 nucleotide long noncoding RNAs that silence target mRNAs for degradation and then inhibiting the translation. miRNAs can target $60 \%$ of mRNAs and control the signalling pathways in most cell types. ${ }^{3}$ ChIP-sequencing combines chromatin immune precipitation 
with massively parallel DNA sequencing to identify the binding sites of DNA-associated proteins. Microbiota investigation is the other important field for disease research; whole microbial genome sequencing reveals the extraordinary diversity of microorganisms on Earth and their vast genetic and metabolic repertoire. The increase in accuracy, length and number of reads generated by high-throughput sequencing has coincided with a surge of interest in the human microbiota, the totality of bacteria associated with the human body, in both health and diseases. ${ }^{4}$

\section{The progress of genomic approaches of asthma, COPD and lung cancer}

Asthma: Asthma affects over 300million people worldwide and is a heterogeneous disease of the airway. ${ }^{5}$ More than one hundred genes were found to have associations with asthma by candidate gene approaches which cannot identify novel pathways. Positional cloning is another approach that identifies disease genes by progressive dissection of linkage regions that are consistently co-inherited with the disease. ADAM33, PHF11, DPP10, GPRA, HLA-G, CYFIP2, $I R K 3, O P N 3 / C H M L$ were discovered as asthma genes by positional cloning. ${ }^{6}$ Most associations identified by positional cloning studies and candidate gene studies have moderate effects in pathogenesis of asthma. The first GWAS study for asthma was carried out in the GABRIEL Consortium. The consortium identified SNPs in the chromosome 17q12-q21 region to be significantly $(\mathrm{P}<10-12)$ associated with childhood asthma and ORM1-like 3 Saccharomyces cerevisiae (ORMDL3) gene. ${ }^{7}$ Then a large consortium GWAS study confirmed $O R M D L 3$ as an important asthma suspected gene. The consortium also found $I L-18 R 1, H L A-D R B I, H L A-D Q, I L-33$, SMAD3, IL-2RB, SLC22A5, IL-13 and RORA as asthma suspected genes. ${ }^{8}$ Since then, more than ten GWASs on asthma have been published. Polymorphisms of Ch13L1, PDE4D, TLE4, ADRA1B, PRNP, DPP10, GNAI3, DENND1B, RAD50, IL-13, HLA-DR-DQ LRP1B, SNX10, CA10, KCNJ2, TSLP, RTP2, PYHIN1, IL-12A, STAT4, IRF2, CDHR3, CTNNA3 and SEMA3D were found to have significant associations with asthma and asthma associated traits. ${ }^{9}, 10$ ORMDL3, GSDMB, TSLP, IL-33, IL-18R1, HLA-D, RAD50, IL-13 are the genes have been identified in multiple GWAS studies of asthma. Human airway epithelium is central to the pathogenesis of asthma. ORMDL3, GSDMB, TSLP and $I L-33$ express in airway epithelium and they may modify the inflammatory response to epithelial damage or regulate transduction pathways that lead to asthma. ORMDL3 influences multiple pathways in the endoplasmic reticulum (ER) that mediate inflammation during asthma. GSDMB may play important roles in cell proliferation. TSLP induces cellular senescence during airway remodelling in asthma and IL-33 is constitutively expressed and is thought to function as an alarmin to alert the immune system after endothelial or epithelial cell damage during trauma or infection.

Transcription profiling also identified interesting suspected genes for asthma. IL-4R was found increased in genome-wide expression profiling in allergic asthma. ${ }^{11}$ IL-5, IL-9 and PRG2 expressed more in response to dust mite allergen interact with environmental in a genome wide differential gene expression study. ${ }^{12}$ To asthma, many environmental factors such as allergens, microbes, tobacco smoke and stress influence epigenetic effects in human cells. Children growing up in a traditional farming environment had lower risk of allergic respiratory diseases. Demethylation of the FOXP3promoter was found in an extensive farming exposure environment. ${ }^{13}$ Farm childhood also had hypomethylation of ORMDL1 and STAT6 and hypermethylation of RAD and IL-13. ${ }^{14}$ DNA methylation in the $C D 14$ promoter was also significantly less in farm mothers. ${ }^{15}$ PBMC s from obese asthmatic children had lower levels of promoter methylation of the CCL5, IL-2RA and TBX21 and higher level promoter methylation of TGFB 1 and FCER $2 .{ }^{16}$ Recent epigenome-wide approach identified 36 loci that had association of serum IgE level. Among them, DNA methylation events have been found in cytokine signalling genes IL-4, IL-5R, transcription factor genes ZNF22, RB1, GATA1, KLF1. ${ }^{17}$ More than 200 miRNAs have been found to associate with asthma. ${ }^{18} \mathrm{miR}-1$, miR-21,miR-126, miR-145 and miR-221 that have potential roles in the development of asthma through the targeting of various cytokines and transcription factors. ${ }^{19}$ For asthma, microbiota investigation found that the bronchial tree contained a characteristic flora that was disturbed by the presence of pathogens such as Haemophilus influenzae..$^{20}$

COPD: Chronic obstructive pulmonary disease (COPD) is a major cause of mortality and morbidity worldwide. ${ }^{21}$ Smoking is the major risk factor for COPD, but only a small portion of individuals develop COPD, indicating the genetic factors underlie the disease pathogenesis. ${ }^{22}$ The most well-known candidate genes for COPD are CHRNA3/5, IREB2, HHIP, FAM13A and AGER. They were replicated and confirmed in multiple populations by GWAS studies. ${ }^{23-25}$ None of them are targeted by treatments for COPD yet, and the mechanisms by which they alter COPD risk are still largely unknown. CHRNA3/5 and CHRNB4 are subunits of the nicotine cholinergic receptor. The cholinergic system is active both in cholinergic neuronal cells and in bronchial epithelial cells. ${ }^{26}$ IREB2 is a protein that binds iron-responsive elements (IREs), and is regulated in response to iron supply and oxygen. The Ireb2 knockout mouse had abnormal iron metabolism and caused cellular dysfunction. ${ }^{27}$ HHIP encodes a membrane glycoprotein for the Hedgehog pathway. Hedgehog signalling is important for the morphogenesis of the lung. ${ }^{28}$ The RhoGAP domain of FAM13A may be related to COPD as Rho GTPases are key regulators of cytoskeletal dynamics, involving in the pulmonary endothelial barrier ${ }^{29}$ AGER is a receptor for advanced glycan end products (RAGE). The receptor belongs to immunoglobulin super family and interacts with molecules implicated in inflammation, and development. ${ }^{30}$ Other candidate genes for COPD include CYP2A6, RAB4B, MIA, EGLN, TGFB2, MMP12, RIN3, GSTDS, GSTCD, INTS12, ADAM19, BICD1 and SERPINE2. ${ }^{26}$ There was little overlap in the genes that underlie asthma and the genes underlie COPD, indicating the two conditions in human airway have different disease pathogenesis.

There was evidence that cigarette smoking is associated with epigenetic in the bronchial epithelium. ${ }^{31}$ Epigenetic studies in lung DNA samples from COPD patients identified the COPD patients have increased AHRR methylation, p16 promoter methylation, Runx 3 methylation, miR-487b methylation, and decreased HDAC6 methylation in lung biopsy from COPD smokers. ${ }^{32}$ In histone modification, COPD patients had increased acetylated $\mathrm{H} 3$ in lung that increased inflammatory gene transcription.$^{33}$ For histone modification enzymes, COPD patients had decrease HDAC2 expression in lung tissues, alveolar macrophage and peripheral blood monocytes. ${ }^{34-36}$ Anti-aging and anti-inflammatory agents SIRT1 and SIRT6 were also found to have decreased in COPD lungs. ${ }^{37,38}$ Currently clinical trials on COPD epigenetic therapy focus on HDACs and the combination therapies including DNA methyltransferases inhibitors and antiinflammatory drugs will provide a promising approach. ${ }^{32} \mathrm{~A}$ few studies identified that cigarette smoking induced a general down-regulation of miRNAs. Significantly lower expression of miR-452 was linked to 
increased expression of metalloproproteinase 12 that was an important effector of smoking related diseases such as COPD, ${ }^{39} \mathrm{MiR}-30 \mathrm{c}$, miR$181 \mathrm{~d}$ and miR-638 may also have role in regional emphysema severity and can modulate genes expression network. miR-368 may play an important roles in COPD pathology by responding to oxidative stress. $^{40} \mathrm{~A}$ recent study for miRNA screening in COPD patients found that miR-218-5p was significantly down-regulated in COPD patients, respective of smoking cessation. Pulmonary expression of miR-218-5p was mainly localized at the bronchial airway epithelium. In vitro and in vivo experiments provided functional data of miR218-5p impacting biological pathways that were relevant to COPD pathogenesis. ${ }^{41}$ The analysis of the bacterial microbiota in COPD lung tissue confirmed that there was a significant difference in composition of the bacterial community between COPD and health lungs. ${ }^{42,43}$ The lungs of patients with very severe COPD contained a different community of bacteria to those of controls. These differences were largely driven by bacteria belonging to either the Proteobacteria or the Firmicutes phylum. ${ }^{42}$

Lung Cancer: Lung cancer can be divided into two broad categories. Small cell lung cancer (SCLC) accounts for $15 \%$ of lung cancer cases and is a highly malignant tumour derived from cells exhibiting neuroendocrine characteristics. Non-small cell lung cancer (NSCLC) is further classified into three major pathologic subtypes: adenocarcinoma, squamous cell carcinoma, and large cell carcinoma. Adenocarcinoma accounts for $38.5 \%$ of all lung cancer cases, with squamous cell carcinoma accounting for $20 \%$ and large cell carcinoma accounting for $2.9 \%{ }^{44}$ There is a genetic component to the pathogenesis of lung cancer, whether it relates to host susceptibility to lung cancer, with or without exposure to cigarette smoke to the development of certain types of lung cancer, or to an individual's responsiveness to biologic therapies. Polymorphisms of CYP1A1, GST, EPHX1, MPO, NQO1, GSTM1, NAT2, XRCC1, OGG1, ERCC1, XPD, XPA, $X R C C 3, p 53, p 73, F A S$ and FASL were found to have association with lung cancer in many studies. ${ }^{45}$ With the environmental stimulation, somatic mutations in genome occur in lung cells all the time. Most of the somatic mutations are harmless, but a mutation affects a gene or regulatory element and causes to a phenotypic consequence. Some of these mutations can confer a selective advantage to the cell, leading to preferential growth or survival of a clone to cancer. Such mutation can work as driver mutation for cancer. ${ }^{46}$ High-throughput DNA sequencing has enabled the systematic sequencing of more than 2500 whole cancer genomes. This has revolutionized our understanding of the genetics of cancer, leading to the discovery of previously unrecognized cancer genes, new mutational signatures, and fresh insights into cancer evolution. ${ }^{46}$ Lung cancer tissues sequencing revealed that mutations of EGFR, KRAS, PIK3CA, and TP53 genes were important mutations for the development of lung cancer. ${ }^{47}$ Next generation sequencing of cell-free circulating tumour DNA make it feasible to fast detection of therapeutically targetable driver and resistance mutations in lung cancer patients. ${ }^{48}$ Acquired or epigenetic changes to DNA chromosome can also lead to increased lung cancer susceptibility.

Despite many genetic association studies, the specific genes responsible for the enhanced risk for lung cancer remain poorly understood. To understand the gene's function, particularly for the driven genes will bring new therapeutic means to treat lung cancer. Selective biological therapies have emerged to effectively treat certain types of cancers or to targetspecific determinants that are expressed by many different cancer types. Adenocarcinoma of the lung is divided according to EGFR mutation, EML4-ALK translocation, $M E T$ amplification, HER2 amplification, FGR4 amplification and KRAS mutation. ${ }^{44}$ The genotypes or expression profiling greatly influence that chemotherapy and targeting therapy of the cancers. Activating mutations in EGFR confer sensitivity to EGFR tyrosine inhibitors, but the secondary mutation $\mathrm{T} 790 \mathrm{M}$ in exon 20 renders inhibitors ineffective. ${ }^{49}$ The most exciting advancement in lung cancer treatment came from immunotherapy. Checkpoint blockade remove the blockade imposed by molecules such as PD1 or PDL1 on checkpoints that are required for $\mathrm{T}$ cell activity. The immunotherapy has proven effective around $25 \%$ of patients. Approaches to increase the response rates and identify new checkpoint molecules are on the way. ${ }^{50}$ Clinical trials for epigenetic therapy to lung cancer are also being carried out worldwide and these investigative therapies also focusing on the epigenetic regulations on DNMTs and HDACs. ${ }^{32}$

\section{The future perspective of personalized medicine for respiratory diseases}

The genetic and genomic approaches of asthma, COPD and lung cancer brought fruitful results for the diseases diagnosis and management in clinic. For the genes that confer the diseases, it is important to understand the functional roles in the diseases. Cellular model, mouse model and translational studies are all necessary tools for identifying the novel genes function and the pathways they regulate in human tissue and cells. Co-immuno precipitation with mass spectrum analysis is also likely to reveal the working partners with the new molecules. Proteomic approach includes liquid chromatography and tandem mass spectrometry (MS/MS) and can be used comprehensively for proteins and their biological function. The pharmacogenomics approach will apply to identify the polymorphisms of the novel genes in the search for novel drug. A knockout mouse is a genetically engineered mouse in which one or more genes have been made inoperative. A conditional knockout approach allows researchers to delete the gene of interest in a time- and space dependent manner. The technique of conditional gene expression applies site-specific DNA recombinase systems in mouse genome. The development of Zinc Finger Nuclease (ZFN) system and clustered regularly interspaced short palindromic repeats (CRISPER) system offer another effective means for genomic editing. Current epigenetic drugs for lung cancer are compounds which have off-target effects and are limiting their options for clinical trials. Single cell sequencing for lung cancer can determine the contributions of intra-tumour genetic heterogeneity in cancer development or treatment response. ${ }^{51}$ Immunotherapy for lung cancer just began. With the development of the genomic technology, it is hoped that in the near future, asthma, COPD and lung cancer will be effectively managed in the clinics with reasonably good medical care.

\section{Acknowledgements}

None.

\section{Conflict of interest}

Author declares that there is no conflict of interest.

\section{References}

1. Siegel R, Ward E, Brawley O, et al. Cancer statistics, 2011: the impact of eliminating socioeconomic and racial disparities on premature cancer deaths. CA Cancer J Clin. 2011;61(4):212-236.

2. Manolio TA. Bringing genome-wide association findings into clinical use. Nat Rev Genet. 2013;14(8):549-558. 
3. Friedman RC, Farh KK, Burge CB, et al. Most mammalian mRNAs are conserved targets of microRNAs. Genome Res. 2009;19(1):92-105.

4. Cox MJ, Cookson WO, Moffatt MF. Sequencing the human microbiome in health and disease. Hum Mol Genet. 2013;22(R1):R88-R94.

5. Masoli M, Fabian D, Holt S, et al. The global burden of asthma: executive summary of the GINA Dissemination Committee report. Allergy. 2004;59(5):469-478.

6. Zhang Y, Moffatt MF, Cookson WO. Genetic and genomic approaches to asthma: new insights for the origins. Curr Opin Pulm Med. 2012;18(1):6-13.

7. Moffatt MF, Kabesch M, Liang L, et al. Genetic variants regulating ORMDL3 expression contribute to the risk of childhood asthma. Nature. 2007;448(7152):470-473.

8. Moffatt MF, Gut IG, Demenais F, et al. A large-scale, consortiumbased genomewide association study of asthma. $N$ Engl $\mathrm{J} \mathrm{Med}$. 2010;363(13):1211-1221.

9. Ober C, Yao TC. The genetics of asthma and allergic disease: a $21 \mathrm{st}$ century perspective. Immunol Rev. 2011;242(1):10-30.

10. Ober C. Asthma genetics in the post-GWAs era. Ann Am Thorac Soc 2016;13(Suppl 1):S85-S90.

11. Pascual M, Roa S, Garcia-Sanchez A, et al. Genome-wide expression profiling of B lymphocytes reveals IL4R increase in allergic asthma. $J$ Allergy Clin Immunol. 2014;134(4):972-975.

12. Sordillo JE, Kelly R, Bunyavanich S, et al. Genome-wide expression profiles identify potential targets for gene-environment interactions in asthma severity. J Allergy Clin Immunol. 2015;136(4):885-892.

13. Schaub B, Liu J, Hoppler S, et al. Maternal farm exposure modulates neonatal immune mechanisms through regulatory T cells. J Allergy Clin Immunol. 2009;123(4):774-782.

14. Michel S, Busato F, Genuneit J, et al. Farm exposure and time trends in early childhood may influence DNA methylation in genes related to asthma and allergy. Allergy. 2013;68(3):355-364

15. Slaats GG, Reinius LE, Alm J, et al. DNA methylation levels within the CD14 promoter region are lower in placentas of mothers living on a farm. Allergy. 2012;67(7):895-903.

16. Rastogi D, Suzuki M, Greally JM. Differential epigenome-wide DNA methylation patterns in childhood obesity-associated asthma. Sci Rep. 2013;3:2164.

17. Liang L, Willis-Owen SA, Laprise C, et al. An epigenome-wide association study of total serum immunoglobulin E concentration. Nature. 2015;520(7549):670-674.

18. Solberg OD, Ostrin EJ, Love MI, et al. Airway epithelial miRNA expression is altered in asthma. Am $J$ Respir Crit Care Med. 2012;186(10):965-974.

19. Booton R, Lindsay MA. Emerging role of MicroRNAs and long noncoding RNAs in respiratory disease. Chest. 2014;146(1):193-204.

20. Hilty M, Burke C, Pedro H, et al. Disordered microbial communities in asthmatic airways. PloS one. 2010;5(1):e8578.

21. Lopez AD, Shibuya K, Rao C, et al. Chronic obstructive pulmonary disease: current burden and future projections. Eur Respir J. 2006;27(2):397-412.

22. Zhou JJ, Cho MH, Castaldi PJ, et al. Heritability of chronic obstructive pulmonary disease and related phenotypes in smokers. Am J Respir Crit Care Med. 2013;188(8):941-947.

23. Pillai SG, Ge D, Zhu G, et al. A genome-wide association study in chronic obstructive pulmonary disease (COPD): identification of two major susceptibility loci. PLoS Genet. 2009;5(3):e1000421.
24. Cho MH, Boutaoui N, Klanderman BJ, et al. Variants in FAM13A are associated with chronic obstructive pulmonary disease. Nat Genet. 2010;42(3):200-202.

25. Chen W, Brehm JM, Manichaikul A, et al. A genome-wide association study of chronic obstructive pulmonary disease in Hispanics. Ann Am Thorac Soc. 2015;12(3):340-348.

26. Kim WJ, Lee SD. Candidate genes for COPD: current evidence and research. Int J Chron Obstruct Pulmon Dis. 2015;10:2249-2255.

27. Zumbrennen-Bullough KB, Becker L, Garrett L, et al. Abnormal brain iron metabolism in Irp2 deficient mice is associated with mild neurological and behavioral impairments. PloS one. 2014;9(6):e98072.

28. Chuang PT, Kawcak T, McMahon AP. Feedback control of mammalian Hedgehog signaling by the Hedgehog-binding protein, Hip1, modulates Fgf signaling during branching morphogenesis of the lung. Genes Dev. $2003 ; 17(3): 342-347$

29. Corvol H, Hodges CA, Drumm ML, et al. Moving beyond genetics: is FAM13A a major biological contributor in lung physiology and chronic lung diseases? J Med Genet. 2014;51(10):646-649.

30. Sorci G, Riuzzi F, Giambanco I, et al. RAGE in tissue homeostasis, repair and regeneration. Biochim Biophys Acta. 2013;1833(1):101-109.

31. Belinsky SA, Palmisano WA, Gilliland FD, et al. Aberrant promoter methylation in bronchial epithelium and sputum from current and former smokers. Cancer Res. 2002;62(8):2370-2377.

32. Schamberger AC, Mise N, Meiners S, et al. Epigenetic mechanisms in COPD: implications for pathogenesis and drug discovery. Expert Opin Drug Discov. 2014;9(6):609-628.

33. Szulakowski P, Crowther AJ, Jimenez LA, et al. The effect of smoking on the transcriptional regulation of lung inflammation in patients with chronic obstructive pulmonary disease. Am J Respir Crit Care Med. 2006;174(1):41-50.

34. Ito $\mathrm{K}$, Ito $\mathrm{M}$, Elliott $\mathrm{WM}$, et al. Decreased histone deacetylase activity in chronic obstructive pulmonary disease. $N$ Engl $\mathrm{J} \mathrm{Med}$. 2005;352(19):1967-1976.

35. Cosio BG, Tsaprouni L, Ito $\mathrm{K}$, et al. Theophylline restores histone deacetylase activity and steroid responses in COPD macrophages. $J$ Exp Med. 2004;200(5):689-695.

36. Mercado N, Thimmulappa R, Thomas CM, et al. Decreased histone deacetylase 2 impairs Nrf2 activation by oxidative stress. Biochem Biophys Res Commun. 2011;406(2):292-298.

37. Rajendrasozhan S, Yang SR, Kinnula VL, et al. SIRT1, an antiinflammatory and antiaging protein, is decreased in lungs of patients with chronic obstructive pulmonary disease. Am J Respir Crit Care Med. 2008;177(8):861-870.

38. Takasaka N, Araya J, Hara H, et al. Autophagy induction by SIRT6 through attenuation of insulin-like growth factor signaling is involved in the regulation of human bronchial epithelial cell senescence. J Immunol. 2014;192(3):958-968.

39. Hunninghake GM, Cho MH, Tesfaigzi Y, et al. MMP12, lung function, and COPD in high-risk populations. N Engl J Med. 2009;361(27):2599-2608.

40. Christenson SA, Brandsma CA, Campbell JD, et al. miR-638 regulates gene expression networks associated with emphysematous lung destruction. Genome Med. 2013;5(12):114.

41. Conickx G, Mestdagh P, Avila Cobos F, et al. MicroRNA Profiling Reveals a Role for MicroRNA-218-5p in the Pathogenesis of Chronic Obstructive Pulmonary Disease. Am J Respir Crit Care Med. 2017;195(1):43-56.

42. Sze MA, Dimitriu PA, Suzuki M, et al. Host Response to the Lung Microbiome in Chronic Obstructive Pulmonary Disease. Am J Respir Crit Care Med. 2015;192(4):438-445. 
43. Huang YJ, Kim E, Cox MJ, et al. A persistent and diverse airway microbiota present during chronic obstructive pulmonary disease exacerbations. OMICS. 2010;14(1):9-59.

44. Herbst RS, Heymach JV, Lippman SM. Lung cancer. $N$ Engl J Med. 2008;359(13):1367-1380.

45. Dela Cruz CS, Tanoue LT, Matthay RA. Lung cancer: epidemiology, etiology, and prevention. Clin Chest Med. 2011;32(4):605-644.

46. Martincorena I, Campbell PJ. Somatic mutation in cancer and normal cells. Science. 2015;349(6255):1483-1489.

47. Feng H, Wang X, Zhang Z, et al. Identification of Genetic Mutations in Human Lung Cancer by Targeted Sequencing. Cancer Inform. 2015;14:83-93.
48. Thompson JC, Yee SS, Troxel AB, et al. Detection of therapeutically targetable driver and resistance mutations in lung cancer patients by next generation sequencing of cell-free circulating tumor DNA. Clin Cancer Res. 2016;22(23):5772-5782

49. Tursz T, Andre F, Lazar V, et al. Implications of personalized medicine-perspective from a cancer center. Nat Rev Clin Oncol 2011;8(3):177-183.

50. Chee J, Robinson BW, Holt RA, et al. Immunotherapy of lung malignancies - from gene sequencing to novel therapies. Chest. 2016.

51. Gawad C, Koh W, Quake SR. Single-cell genome sequencing: current state of the science. Nat Rev Genet. 2016;17(3):175-188. 